\title{
Supplementary Information: Latent Representation Learning for Structural Characterization of Catalysts
}

Prahlad K. Routh ${ }^{1 \dagger}$,Yang Liu ${ }^{1 \dagger}$, Nicholas Marcella ${ }^{1 \dagger}$, Boris Kozinsky ${ }^{2,3}$, Anatoly I. Frenkel ${ }^{1,4^{*}}$

${ }^{1}$ Department of Materials Science and Chemical Engineering, Stony Brook University, Stony

Brook, NY 11794, USA

${ }^{2}$ John A. Paulson School of Engineering and Applied Sciences, Harvard University, Cambridge, MA 02138, USA

${ }^{3}$ Bosch Research, Cambridge, MA 02139, USA

${ }^{4}$ Division of Chemistry, Brookhaven National Laboratory, Upton, NY 11973, USA

$\dagger$ Equally Contributing Authors

Corresponding Author

* Email address: anatoly.frenkel@stonybrook.edu 


\section{S1: Theoretical XANES of Pd atomic clusters}

Simulation of XANES for the Pd atomic clusters: Four Pd atomic clusters were constructed with the nearest Pd-Pd bond length varied between $2.456 \AA$ and $3.002 \AA$. The structure of the Pd clusters were shown in the Figure $\mathrm{S} 1$. The center atom was selected as absorbing atom. $5000 \mathrm{Pd}_{2}, 2156 \mathrm{Pd}_{3}, 5000 \mathrm{Pd}_{4}, 5000 \mathrm{Pd}_{5}$ clusters were constructed for FEFF9 simulation. The non-structural parameters for XANES simulations were chosen to ensure the best agreement between the simulated spectrum for bulk Pd and the corresponding experimental XANES data. FEFF version 9.9.1 was used for self-consistent calculations within full multiple scattering (FMS) and muffin-tin (MT) approximations. For Pd K-edge simulations, we use the complex Hedin-Lundqvist (HL) exchange correlation potential, cluster with radius $5.5 \AA$ for self-consistent field (SCF) calculations, cluster of radius of $7 \AA$ for FMS calculations, and $\mathrm{S}_{0}^{2}$ of 0.9 , where the core-hole is treated with the random phase approximation (RPA). The RMULTIPLIER card was used to modify the lattice constant to obtain those listed in the previous paragraph.

Autoencoder for Pd atomic clusters: All the theoretical XANES from FEFF are interpolated on the same energy scale ranging from $E_{\min }=24370.9 \mathrm{eV}$ to $\mathrm{E}_{\max }=24451.1 \mathrm{eV}$. Then each spectrum was represented as 98 points of absorption coefficients which are treated as input/output and are selected to train and test (with a $10 \%$ held out data) the autoencoder with varied number of nodes in latent space. The encoder part has two linear layers with 500 nodes and decoder part also has two linear layers with 500 nodes. Each layer has been followed with ReLU activation except for the last layer. The mean squared loss has been used as the error metric in the neural network training process to get the best neural network.

Reconstruction Error: Box-whisker plot denotes a box with median (50th percentile), first quartile (25th percentile) and third quartile (75th percentile) of the MSE loss along with the outliers (beyond 1.5 times of interquartile range) as dots. The whiskers denote the minimum and maximum values after excluding the

outliers. Figure S1 shows the change in reconstruction loss (mean squared error between original spectra and reconstructed spectra in the test dataset) with increase in the number of bottleneck nodes. 500 datapoints 
were used for a, c, d and 216 datapoints for b to calculate the MSE loss. Figure S2 shows the agreement between input XANES and its reconstruction for $\mathrm{Pd}_{2}$ and $\mathrm{Pd}_{3}$ atomic clusters.
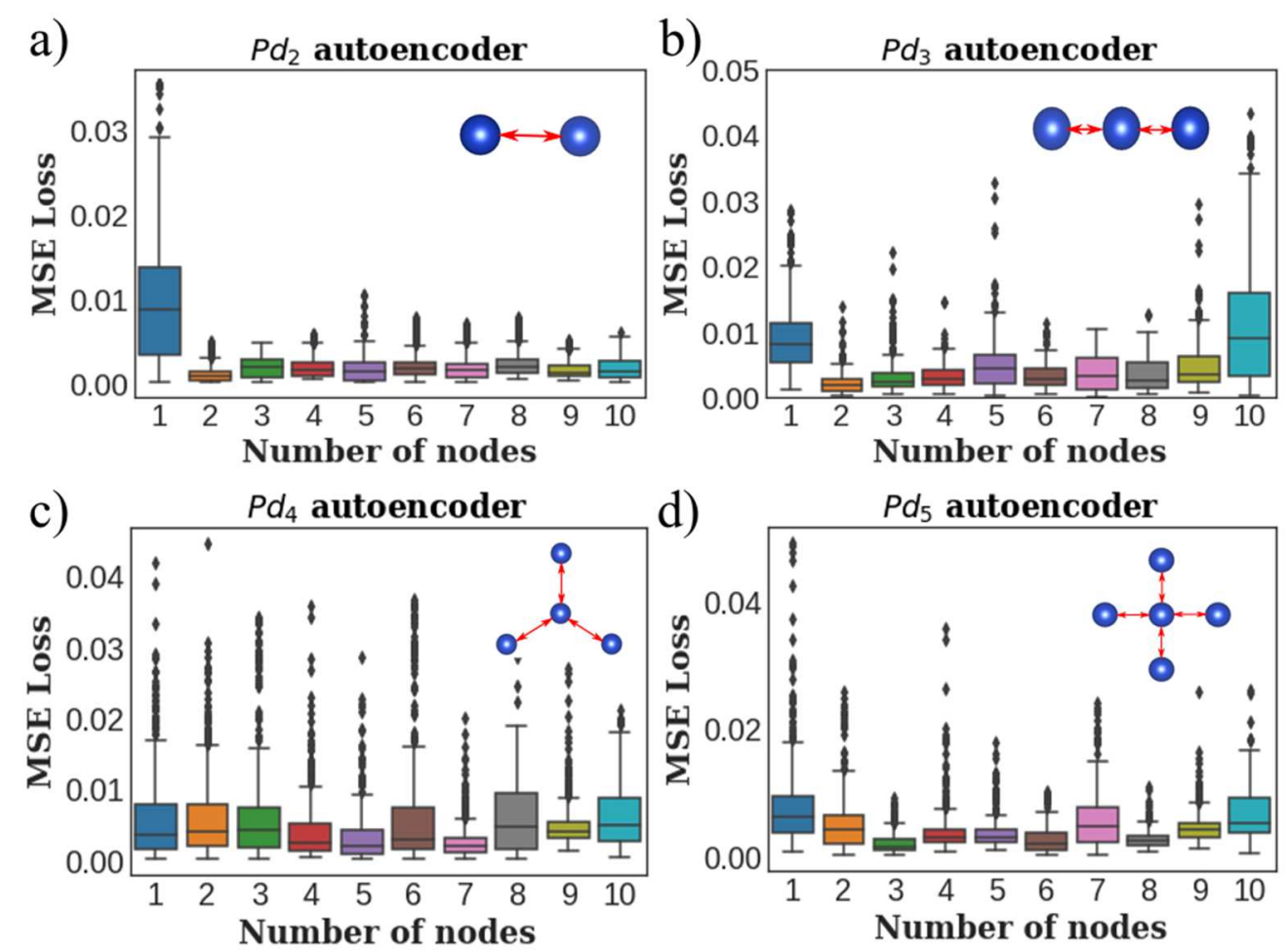

Figure S1: Box whisker plot of MSE Loss vs the number of nodes in latent space showing the reconstruction performance of autoencoders for the Pd atomic clusters composed of a) 2, b) 3, c) 4, and d) 5 atoms. 


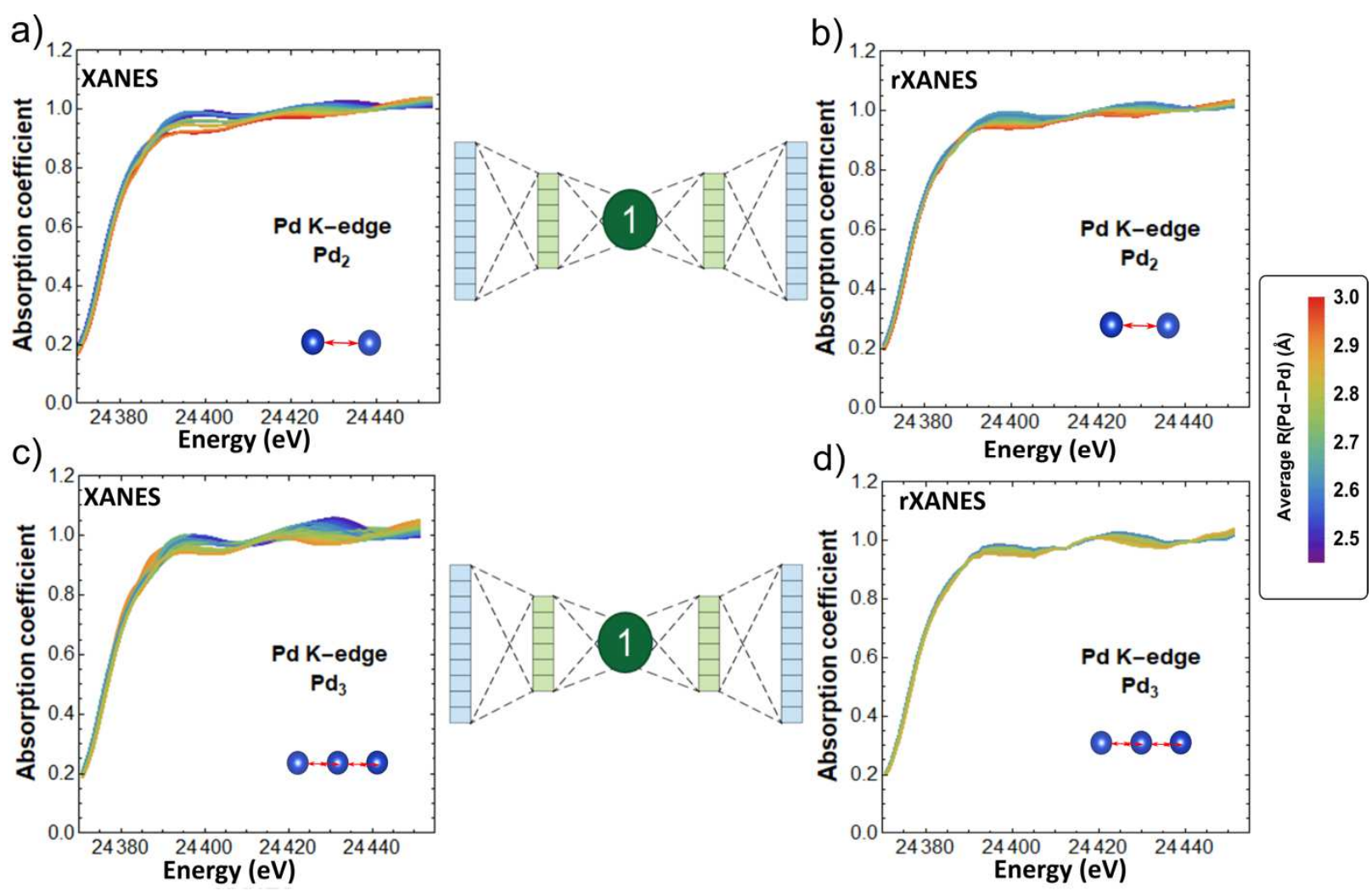

Figure S2: The comparison of the XANES of $\mathrm{Pd}_{2}, \mathrm{Pd}_{3}(\mathrm{a}, \mathrm{c})$ with the reconstructed XANES spectra (b, d) through the autoencoder where the bottleneck layer has one node.

\section{S2: Theoretical XANES of Pd nanoparticles}

Simulation of XANES for Pd nanoparticles and training dataset generation: 26 atomistic Pd NP motifs of different packing (FCC, HCP, and OCT) and particle sizes (6 to 153 atoms) were generated for lattice constants 3.801, 3.842, 3.882, 3.923, 3.963, 4.004, 4.044, and $4.125 \AA$, resulting in 208 Pd NP models. The symmetrically unique sites were selected, resulting in 1253 unique Pd atom sites. For each $\mathrm{Pd}$ atom site, the total number of octahedral sites in the first nearest neighbor shell was determined and defined as $h_{\max }$. For each Pd site, in a sequential manner, a hydrogen atom was added to a randomly selected octahedral site, resulting in a new structure with a hydrogen occupancy $h_{\text {frac }}$ defined by the number of added hydrogens, 
$N_{h}$, dived by $h_{\max }$, i.e. $h_{f r a c}=N_{h} / h_{\max }$. This procedure resulted in $h_{f r a c}$ of $0,0.1667,0.2,0.25,0.3333$, $0.4,0.5,0.6,0.6667,0.75,0.8,0.8333$, and 1 . The total number of training examples was then 5,565 .

As in our previous work, ${ }^{1-5}$ we used FEFF9 ${ }^{6}$ for XANES simulations. The non-structural parameters for XANES simulations were chosen to ensure the best agreement between the simulated spectrum for bulk Pd and the corresponding experimental XANES data. FEFF version 9.9.1 was used for self-consistent calculations within full multiple scattering (FMS) and muffin-tin (MT) approximations. For Pd K-edge simulations, we use the complex Hedin-Lundqvist (HL) exchange correlation potential, cluster with radius $5.5 \AA$ for self-consistent field (SCF) calculations, cluster of radius of $7 \AA$ for FMS calculations, and S02 of 0.9, where the core-hole is treated with the random phase approximation (RPA). The RMULTIPLIER card was used to modify the lattice constant to obtain those listed in the previous paragraph.

To maximize diversity, and increase the size of our training data set, we trained the NNs on linear combinations of the 5,565 site-specific data. This approach, introduced in our previous works, ${ }^{3}$ mimics the particle-averaging effect in experimental XANES data, and takes advantage of the fact that particle-average XANES $\mu(E)$ and coordination number $N_{P d}$ are linear combinations of the XANES spectra and coordination numbers, respectively, calculated for each absorbing site $j$ :

$$
\begin{gathered}
\mu(E)=\frac{\sum_{j} \mu_{j}(E)}{N_{a}}, \\
\text { and } N_{P d}=\frac{\sum_{j} C_{P d}^{j}}{N_{a}} .
\end{gathered}
$$

Therefore, using a relatively small set of XANES-CNs pairs obtained for individual sites, we can generate large, diverse, numbers of labeled examples for the $\mathrm{NN}$ training set. For $\mathrm{NN}$ training, we use random linear combinations of 3 XANES-CNs pairs from the pool of site-specific calculations, with replacement, thus approximately $3 \times 10^{10}$ possible synthetic training examples can be generated without repetition. 
Autoencoder for theoretical Pd nanoparticles: After the FEFF simulation and data processing of the Pd nanoparticles as described in section S2, all of the theoretical XANES are interpolated on the same energy scale ranging from $\mathrm{Emin}=24339.1 \mathrm{eV}$ to $\mathrm{Emax}=24421.8 \mathrm{eV}$. Then each spectrum was represented as 100 points of absorption coefficients which are treated as input/output and are selected to train and test (with $10 \%$ held out data) the autoencoder with varied number of nodes in latent space. The encoder part has two linear layers with 500 nodes and decoder part also has two linear layers with 500 nodes. Each layer has been followed with ReLU activation except for the last layer. The mean square loss has been used as the error metric in the neural network training process to get the best neural network.

Mapping of Latent Space to descriptors: The autoencoder with the bottleneck layer of six was selected because of the minima observed in MSE loss plot in Figure 3c and Figure S3. The trained encoder part was used to obtain compressed latent representation of the theoretical XANES of Pd nanoparticles. Furthermore, in order to map, we use this latent space as the input and the real descriptors (coordination number (Pd-Pd), interatomic distance (Pd-Pd) and hydrogen fraction) as output. 10\% of the dataset was held out as test set. The architecture of the neural network for the training is two linear layers with 100 nodes each layer. The ReLU activation followed each layer except output layer. The mean square loss has been used as the error metric in the NN training process to get the best neural network. 


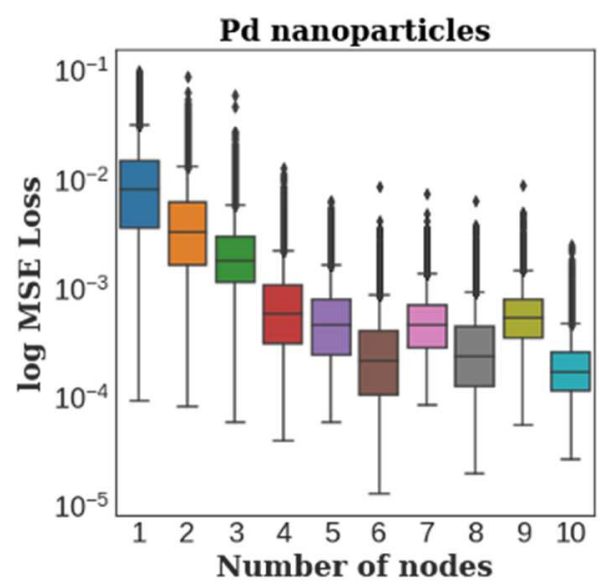

Figure S3: Semi-log scale box-whisker plot of reconstruction loss (MSE Loss) for autoencoder networks with increasing number of nodes in bottleneck layer. Box denotes median and interquartile range and whiskers denote the minimum and maximum values along with the outliers as dots. The test dataset contained 9400 spectra.

Calculation of Correlation Map: The Pearson correlation coefficients between latent space and the descriptors $(\mathrm{N}, \mathrm{R}, \mathrm{H})$ were calculated using Python's NumPy package and the output is noted in Figures 4 and 5. All non-zero correlation coefficients were also tested for statistical significance and a two-tailed pvalue test. The largest p-value was found to be 0.0042 (Figure 5) showing statistically significant results.

\section{S3: Experimental XANES of Pd nanoparticles}

Theoretical XANES of Pd nanoparticles from Section S2 are interpolated on the same energy scale ranging from $\mathrm{E} \min =24339.1 \mathrm{eV}$ to $\mathrm{E} \max =24421.8 \mathrm{eV}$. Input layer in the encoder was followed by 3 convolutions layers and average pooling layers between each. The output of convolutional layers was flattened and followed by a linear layer of 512 nodes before going through a bottleneck of variable length. 15 autoencoders were trained with bottle neck lengths 1-15 (D1-D15). Decoder parameters were similar to the encoder and are summarized in tabular format in Figure S4. 
The MSE loss was taken between the input XANES and reconstructed XANES (rXANES) every batch of 64 examples. The training data was as described above, and a validation loss function was taken for a set of 1000 examples not used in training. The MSE loss of the validation set minimized after 7 bottleneck nodes (Fig. 7c). The 15 trained autoencoders were also used to reconstruct the experimental data, Pd NPs on $\mathrm{Al}_{2} \mathrm{O}_{3}$, described in main text and previously published work ${ }^{7}$. The reconstruction loss (MSE loss) between the input experimental XANES and rXANES was also minimized at 7 nodes (Fig 7f). Visually, the experimental XANES shows good agreement with rXANES (Fig. 7a-b, 7d-e).

\begin{tabular}{|c|c|c|c|c|c|c|c|c|c|c|c|c|c|}
\hline & Convolution & |Pooling | & & Sonvolution & |Pooling & Convolution & Pooling & & Flatten & & Linear & & Linear \\
\hline Input & $1 \times 100$ & $8 \times 100$ & & $8 \times 98$ & $16 \times 50$ & $16 \times 24$ & $32 \times 11$ & Input & $32 \times 3$ & Input & 96 & Input & 512 \\
\hline Kernel & $\{3\}$ & $\{3\}$ & & $\{2\}$ & $\{3\}$ & $\{6\}$ & $\{3\}$ & & & Activation & $\begin{array}{c}\text { Parametric } \\
\text { ReLU }\end{array}$ & Activation & $\begin{array}{c}\text { Parametric } \\
\text { ReLU }\end{array}$ \\
\hline Stride & $\{1\}$ & $\{1\}$ & & $\{2\}$ & $\{2\}$ & $\{2\}$ & $\{3\}$ & & & & & & \\
\hline Padding & $\{\{1,1\}\}$ & $\{\{0,0\}\}$ & & $\{\{1,1\}\}$ & $\{\{0,0\}\}$ & $\{\{1,1\}\}$ & $\{\{0,0\}\}$ & & & & & & \\
\hline Dilation & $\{1\}$ & & & $\{1\}$ & & $\{1\}$ & & & & & & & \\
\hline \multirow[t]{2}{*}{ Activation } & $\begin{array}{c}\text { Parametric } \\
\text { ReLU }\end{array}$ & Mean & & $\begin{array}{c}\text { Parametric } \\
\text { ReLU }\end{array}$ & Mean & $\begin{array}{c}\text { Parametric } \\
\text { ReLU }\end{array}$ & Mean & & & & & & \\
\hline & $8 \times 100$ & $8 \times 98$ & & $16 \times 50$ & $16 \times 24$ & $32 \times 11$ & $32 \times 3$ & Output & 96 & Output & 512 & Output & 7 \\
\hline & & Linear & & Linear & & Reshape & & \multicolumn{2}{|c|}{ Deconvolution } & \multicolumn{2}{|c|}{ Deconvolution } & \multicolumn{2}{|c|}{ Deconvolution } \\
\hline & Input & 7 & Input & 512 & Input & 96 & Input & \multicolumn{2}{|c|}{$32 \times 3$} & \multicolumn{2}{|c|}{$16 \times 10$} & \multicolumn{2}{|c|}{$8 \times 46$} \\
\hline & \multirow[t]{4}{*}{ Activation } & ReLU & Activation & ReLU & Output & $32 \times 3$ & Kernel & \multicolumn{2}{|c|}{6} & \multicolumn{2}{|c|}{3} & \multicolumn{2}{|l|}{10} \\
\hline & & & & & & & Stride & \multicolumn{2}{|c|}{2} & \multicolumn{2}{|c|}{5} & \multicolumn{2}{|l|}{2} \\
\hline & & & & & & & Padding & \multicolumn{2}{|c|}{$\{0,0\}$} & \multicolumn{2}{|c|}{$\{1,1\}$} & \multicolumn{2}{|l|}{$\{0,0\}$} \\
\hline & & & & & & & Activation & \multicolumn{2}{|c|}{ ReLU } & \multicolumn{2}{|c|}{ ReLU } & \multicolumn{2}{|l|}{ ReLU } \\
\hline & Output & 512 & Output & 96 & & & Output & \multicolumn{2}{|c|}{$16 \times 10$} & \multicolumn{2}{|c|}{$8 \times 46$} & $1 \times 100$ & \\
\hline
\end{tabular}

Figure S4: Pd nanoparticle specific NN architecture as implemented in Mathematica 12. The layers are connected sequentially.

Similar to the last section with the D6 autoencoder for theoretical XANES (Fig. 5), we also probe the latent space of the D7 autoencoder using the PCA method (Fig. 8). A set of theoretical data was constructed with varied coordination number $\mathrm{N}$, interatomic distance $\mathrm{R}$, and hydrogen fraction $h_{\text {frac }}$, and constant number of atoms and packing motif (55 atoms, FCC). The variance of the LTLS indicated 3-4 important sources of variance within the data, which is what we expect for this data set. The results of LTLS correlation indicate the first 3 variances are correlated with $\mathrm{N}, \mathrm{R}$, and $h_{\text {frac }}$. 
D7 LTLS was then used to probe the experimental data (Fig. 8a). The variance of the LTLS indicated 1 to 2 important sources of variance within the data. Because we have the EXAFS-derived interatomic distances from Ref. 7, we were able to test the correlation of the distances with the LTLS. As expected, the first variance of the LTLS was highly correlated with R.

Mapping of experimentally obtained XANES to physical descriptors: The D7 latent space of the experimental data contains information regarding the interatomic distance, as determined in the last section. In order to invert the information to physical descriptors we design a MLP to map the latent space to interatomic distance $(\mathrm{R})$. The MLP uses the same training data as the autoencoders, but it is trained in a supervised manner using with latent space as input and $\mathrm{R}$ as the output rather than $\mathrm{rXANES}$. The parameters of MLP used for this mapping are described in Fig. S5. The results of D7 conversion agree very well with the interatomic distances obtained via EXAFS fitting (Fig. 8b).

\begin{tabular}{|c|c|c|c|}
\hline & Linear & Linear & Linear \\
\hline Input & 7 & 7 & 33 \\
\hline Activation & none & ReLU & none \\
\hline Output & 7 & 33 & 1 \\
\hline
\end{tabular}

Figure S5: Pd nanoparticle specific MLP for mapping descriptors from low the encoder output as implemented in Mathematica 12. The layers are connected sequentially. 


\section{References}

(1) Marcella, N.; Liu, Y.; Timoshenko, J.; Guan, E.; Luneau, M.; Shirman, T.; Plonka, A. M.; van der Hoeven, J. E. S.; Aizenberg, J.; Friend, C. M.; et al. Neural Network Assisted Analysis of Bimetallic Nanocatalysts Using X-Ray Absorption near Edge Structure Spectroscopy. Phys. Chem. Chem. Phys. 2020, 22, 18902-18910.

(2) Liu, Y.; Marcella, N.; Timoshenko, J.; Halder, A.; Yang, B.; Kolipaka, L.; Pellin, M. J.; Seifert, S.; Vajda, S.; Liu, P.; et al. Mapping XANES Spectra on Structural Descriptors of Copper Oxide Clusters Using Supervised Machine Learning. J. Chem. Phys. 2019, 151, 164201.

(3) Timoshenko, J.; Lu, D.; Lin, Y.; Frenkel, A. I. Supervised Machine-Learning-Based Determination of Three-Dimensional Structure of Metallic Nanoparticles. J. Phys. Chem. Lett. 2017, 8, 5091-5098.

(4) Timoshenko, J.; Frenkel, A. I. "Inverting" X-Ray Absorption Spectra of Catalysts by Machine Learning in Search for Activity Descriptors. ACS Catal. 2019, 9, 10192-10211.

(5) Timoshenko, J.; Halder, A.; Yang, B.; Seifert, S.; Pellin, M. J.; Vajda, S.; Frenkel, A. I. Subnanometer Substructures in Nanoassemblies Formed from Clusters under a Reactive Atmosphere Revealed Using Machine Learning. J. Phys. Chem. C 2018, 122, 21686-21693.

(6) Rehr, J. J.; Kas, J. J.; Vila, F. D.; Prange, M. P.; Jorissen, K. Parameter-Free Calculations of X-Ray Spectra with FEFF9. Phys. Chem. Chem. Phys. 2010, 12, 5503-5513.

(7) Wang, J.; Wang, Q.; Jiang, X.; Liu, Z.; Yang, W.; Frenkel, A. I. Determination of Nanoparticle Size by Measuring the Metal-Metal Bond Length: The Case of Palladium Hydride. J. Phys. Chem. C 2015, 119, 854-861. 